\title{
A Tool for the Semantic Analysis and Recommendation of Videos in e-Learning
}

\author{
Mauro Coccoli and Gianni Vercelli \\ DIBRIS \\ University of Genoa \\ Genoa, Italy \\ \{mauro.coccoli, gianni.vercelli\}@unige.it
}

\begin{abstract}
Video lessons are increasingly adopted in education, especially in universities and lifelong learning projects. Their popularity is due to the people's familiarity with video and to other intrinsic characteristics of this medium, such as the message rapidity and its reproducibility. Accordingly, Massive Open Online Courses are gaining a prominent role in both formal and informal education and many universities provide video courses for their students through suited platforms or even freely accessible to everyone. To improve the effectiveness of video lessons and to make them part of a wider learning environment, we decided to investigate the possibility of making a system that, starting from a video, can suggest further "readings". Such a system is thought for independent lifelong learners, for regular students, for teachers and instructional designers as well.
\end{abstract}

Keywords-Cognitive computing; video lessons; MOOCs; technology enhanced learning; lifelong learning.

\section{INTRODUCTION}

The use of video lessons is becoming more and more popular among students for a variety of reasons. Since the raise of specialized portals such as, e.g., Vimeo and YouTube, a huge number of tutorials and howtos were made available spontaneously by the users in the form of video, which cover almost any topic, ranging from, e.g., makeup to housekeeping and cars or bikes maintenance. This new wave of User Generated Contents (UGC) dramatically changed the use of the Web, the Internet traffic profiles, and the approach of people towards learning. In fact, people started looking for videos instead of text to clarify concepts, see examples, find additional information on the topic they are interested in or are studying. Yet, there is a lack of organization in these gigantic, general purpose video repositories. Especially, it is difficult to obtain information on the selected resources and get details on their content, hence accessing relevant video fragments is a hard task. Considering the fact that YouTube is worldwide the second largest source of information on the Internet after Google [1], the lack of a correct cognitive alignment between the available videos and the expected learning outcomes with respect to the given audience is still an open problem. To cope with this problem, a semantic support would be required, to enable the effective use of metadata and to improve research capabilities. Unfortunately, in most cases, videos that can be found on the Internet are user-generated and self-produced and we cannot expect a consistent behavior from such a very large, heterogeneous group of persons. Then, alternative solutions must be found so that the needed information can be extracted from videos through automated tools and sophisticated mining techniques.

It is worthwhile noticing that teachers are directly involved in this revolution both as actors and directors. Many of them are producing video courses for enhancing their traditional classes and/or for creating new educational projects in MOOCs (Massive Open Online Courses) platforms, whose number is fast-growing. In fact, following the wave of the OpenCourseWare initiative launched by the Massachusetts Institute of Technology (MIT) and the success of services such as, e.g., Coursera, many universities decided to counteract this phenomenon and provide own video courses through internal and national portals. It is the case of national French platform FUN (France Université Numérique), based on the edX platform, and of the recently released Eduopen initiative in Italy, which sees the authors of this paper directly involved in the production of video lessons for the "Computer Science" modules.

Beside their popularity, video lessons are to be considered an effective also from the learning point of view, for they offer the following advantages:

(i) enhance engagement,

(ii) improve communication,

(iii) improve clarity,

(iv) show examples,

(v) offer the possibility of using captions and exploiting videos recorded in different languages,

(vi) introduce interactivity elements,

(vii) make educational resources freely accessible with reduced costs for development and delivery [2].

Given these premises, we forecast a near future in which it will be easier and easier to find videos on the Web covering a wide variety of topics, also in academic fields, that can be considered reliable due the prestige of the authors and to the presence of metadata qualifying their content and authors as well. Hence, in this paper, we showcase a demonstrative prototype recently developed, which was designed to perform the following activities: 
(i) find interesting videos over the Web on a given matter,

(ii) play videos,

(iii) make a real-time transcript of the video, which can be used for other activities such as collecting text for annotation and semi-automatic translation from foreign language to individuals' mother language,

(iv) process the resulting text with advanced semantic algorithms to extract concepts and relevant information and to find related material in the form of more videos, additional readings, link to other learning assets.

We highlight that the presented prototype is based on the use of cognitive computing solutions and a cloud infrastructure, whose technical details are depicted in the following.

The remainder of the paper is organized as follows. Firstly, in Section 2, an overview of the reasons for using videos in education is given, also covering issues related to the adoption of MOOCs. Section 3 describes new opportunities offered by cognitive computing and how they can be profitably exploited in education. Accordingly, Section 4 provides a detailed description of the proposed system that aims to be a valuable support to both teachers and learners. Finally, Section 5 summarizes findings and gives a glance on future works.

\section{USING VIDEOS IN E-LEARNING AND MOOCS}

In the last five years, MOOCs have emerged as a worldwide adopted, low-cost e-learning technology to deliver video-based educational contents on a wide variety of topics. Given the lack of entry barriers (prerequisites), a lot of universities offers platforms and facilities for their students to enroll in MOOCs. However, only a small fraction of enrolled students actively participates in the various activities, such as viewing video lectures, completing quizzes and homework, and so on. In this respect, the dropout phenomenon has been studied [3], [4]. Several researchers have analyzed usage logs to determine the factors associated with MOOCs' high attrition rate and to develop methods that predict how participants will perform and whether they are likely to drop out [5]. This study shows the results of a research focused on the perception of students about learning in a cooperative MOOC course. It is a university MOOC course, since it is inserted into the formal teaching of Master Degree in Primary Education, although open to participation by all interested persons. The perception of students is evaluated through the questionnaire Technology Acceptance Model (TAM) adapted to the context of learning in a massive open online course. Three variables associated with the perception of students on learning in the course are discussed:

(i) the academic level of participants (undergraduate vs. nondegree students),

(ii) the type of participation in the course (hidden, moderately hidden, active, individualistic and collaborator),

(iii) the gender variable.

\footnotetext{
${ }^{1}$ Borrowed from https://console.ng.bluemix.net/catalog - Can be subject to modifications.
}

Although the perception of the students in income, motivation and ease of use of a MOOC course is high, there are some differences between the variables analyzed. It is noted that no grade students perceive more positively the methodology with which one has to work in a MOOC. However, the type of participation does not significantly influence the perception of students about learning in a MOOC course. In addition, males scored significantly higher on both the motivation to learn through a MOOC, and the perception of its usefulness for learning. The results show good acceptance by students and the use of massive open online courses in regulated university contexts, and suggest a greater acceptance in learning environments linked to lifelong learning and professional development.

\section{Semantics And Cognitive Computing}

The above-cited tool, whose details will be duly discussed in Section 4, relies on sophisticated cognitive computing techniques made available through the rich set of services and APIs accessible within the IBM Bluemix platform [6]. Owing to the IBM Academic Initiative, universities can freely experiment Watson and other functionalities. In particular, the platform provides programmers with a plenty of services based on the Watson artificial intelligence [7] and the DeepQ\&A algorithm, enabling the creation of complex applications and, thus, giving a significant contribution to the increasing success of cognitive computing applications in many fields of applications, including university classes [8]. In more detail, Watson is a full-featured cognitive computing system for the research and development of cognitive systems and services [9], [10], which can easily interoperate with other applications and legacy systems as well. It is a valuable commercial tool, exposing a varied catalog of building blocks, which allow rapid prototyping of applications with advanced features. For example, among the others, we cite the following boilerplates ${ }^{1}$ that can help enhance, scale, and accelerate human expertise in the direction of making software applications smarter and smarter:

(i) Concept Expansion - performs text analysis and can learn similar terms as well as words or phrases, based on context. Such a tool enables users to rapidly create a lexicon and a set of related terms from data sets of text fragments or collections of documents. Then, the output can be used to provide further understanding of data and improve text analytics pipelines;

(ii) Concept Insights - looks for associations of concepts inside sets of documents provided by users with a pre-existing graph of concepts based on the renowned free encyclopedia Wikipedia. Accordingly, the service identifies links of two types: explicit links in the case a document directly mentions a concept, and implicit links, which connect the input documents to relevant concepts that are not directly mentioned. This service can also search for documents that are relevant to a concept or collection of concepts by exploring both the explicit and the implicit links;

(iii) Dialog - allows developers designing the interaction mechanisms of an application with an end-user, based on a 
natural conversational interface. In practice, this service enables computer applications to use natural language and this capability can be profitably exploited in a variety of situations such as, e.g., automatically respond to user questions, walk users through processes or applications, or even hand-hold users through difficult tasks;

(iv) Natural Language Classifier - applies cognitive computing techniques to analyze sentences or phrases in a given corpus and return the best matching classes;

(v) Relationship Extraction - parses sentences into their various components, looking for relationships between the components;

(vi) Speech-to-text and Text-to-speech - the former converts the human voice into the written word and the latter processes text and natural language to generate synthesized audio output complete with appropriate cadence and intonation even if on a limited set of languages;

(vii) Tone Analyzer - leverages cognitive linguistic analysis to identify tones that people show in their languages;

(viii) Visual Recognition - allows analyzing the visual appearance of images or video frames to understand what is happening in a scene. Based on machine learning technology, the semantic classifiers recognize many visual entities, such as, e.g., settings, objects, and events;

(ix) Tradeoff Analytics - helps people make better choices while taking into account multiple, often conflicting, goals that matter when making that choice.

Owing to such an easy-to-use arsenal of services with stunning functionalities, new opportunities arise for both designers and programmers, which can create unprecedented solutions. To display publicly all of its power and potential, IBM Watson attended the competition of the Jeopardy! Game in which it was able to show its ability [11], [12] and [13]. In fact, Watson was victorious and this is due to its ability in finding answers to questions in an unlimited domain. The Watson algorithms can mine both structured and unstructured data, extracting information and originating new knowledge, which results in the emergence of multiple answers that are weighted through a confidence estimation. The key feature required by the competition was mastering the language [14] because the game proposes clues full of irony, subtle meanings, and other complexities allowed by a malicious use of natural language [15]. For what concerns the underlying technology, we report that Watson owes its smartness to the DeepQA Project [16] a massively parallel probabilistic evidence-based architecture, which can also be adapted to different business applications and additional exploratory challenge problems including medicine, enterprise search, and gaming [17].

To conclude this short overview on cognitive computing capabilities, we highlight that the exploitation of cognitive computing services is education is a paramount field of application. Specifically, according to the smarter university model [18], cognitive computing-based applications and services should be adopted for administration and management, and learning activities as well. From the e-learning point of view, we report some experiences that witness how cognitive computing can be an accelerator for students' achievements, and a valuable support for the teachers. In particular:

(i) integrating cognitive computing services in software applications can strongly enhance students' performances in computer science classes;

(ii) studying cognitive computing behavior can lead to significant results in Artificial Intelligence (AI) related studies;

(iii) using a cognitive computing layer for digital interactions with students can enhance their performances and ease the teachers' job in managing both classes and learning materials.

The interested readers can find a comprehensive review of cognitive computing in education in reference [19]. Moreover, cognitive computing techniques allow managing big data and these two issues mixed together are influencing the learning process [20].

\section{THE Video ANALYSIS AND RECOMMENDATION SYSTEM}

To the aim of testing the effectiveness of cognitive computing techniques in the application of semantics, the demonstrative prototype that we are going to describe was made exploiting videos from the course "Computer Science 101", which are made freely available on the website of the Stanford University through their Stanford openEdX e-learning environment. We have chosen this course due the authority of the provider and to the quality of the spoken English, since we want the automated systems to perform speech-to-text conversion. From a very technical point of view, the service is made through the pipelining of different services borrowed from the Watson catalog, according to the sequence shown by the block diagram depicted in Figure 1.

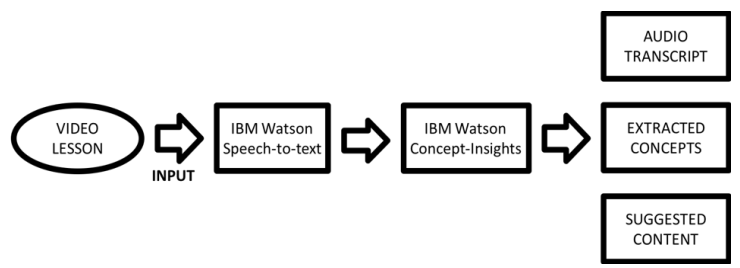

Figure 1: the sequence of operations

More precisely:

(i) a wide variety of videos is presented to the users;

(ii) the user is requested to select a specific video, which starts playing within the browser;

(iii) the video flows through a speech-to-text analysis, which generates its transcript;

(iv) the transcript outcome of the previous module flows through the Concept Insights module, which links the provided documents with a pre-existing graph of concepts based on Wikipedia;

(v) two types of links are identified: explicit links when a document directly mentions a concept, and implicit links which 
connect your documents to relevant concepts that are not directly mentioned in them;

(vi) users can search for documents that are relevant to a concept or collection of concepts by exploring the explicit and implicit links;

(vii) the concepts identified in (iv) are ordered on the basis of the confidence level; user.

(viii) other related videos or web pages are suggested to the

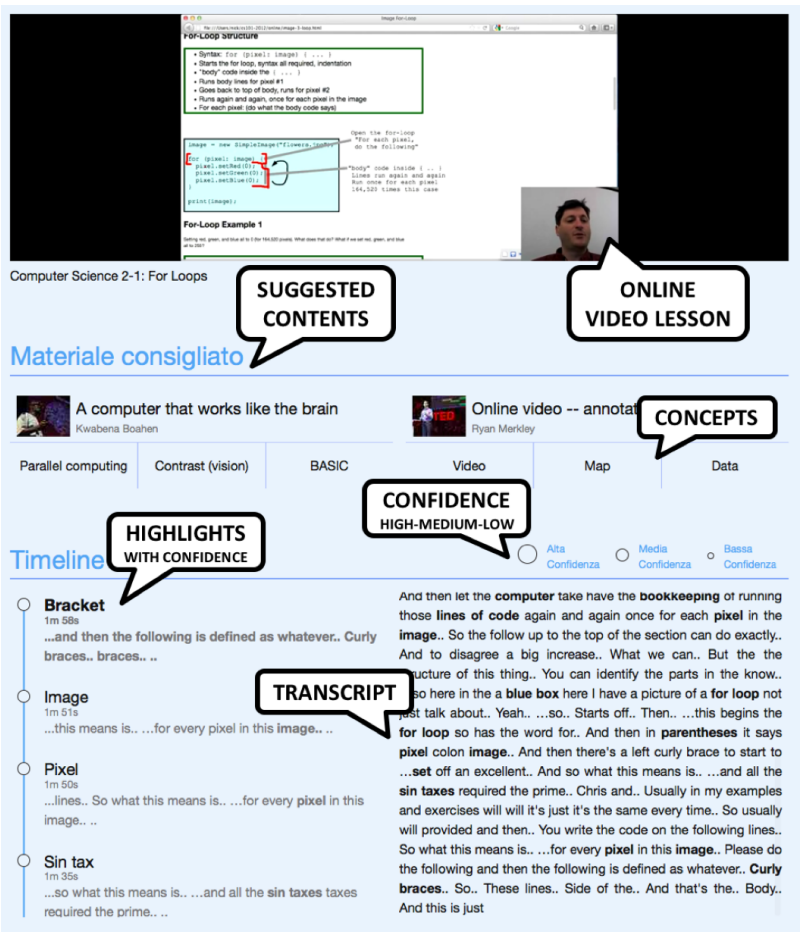

Figure 2: screenshot of the application while running, performing real-time transcript of text and the relevant semantic analysis, resulting in the suggested contents balloon

As a final result, data collected and originated by the analysis are presented in a single web page. This includes (see Fig. 2):

(i) the transcript of the video,

(ii) a timeline with a real-time overview of the keywords identified while the video plays,

(iii) a confidence estimation,

(iv) a list of the extracted concepts, and

(v) suggested related contents.

It is worthwhile noticing that these features can be useful also from an instructional designer point of view [21], which could make a video speaking about a topic, which will become the equivalent of the research keywords in standard search engines. In this way, basic concepts will be extracted from the video and these will become the table of contents. At the same time, the system will look for other related videos, which could enhance student engagement through extra credits (using gamification techniques [22] [23]) be adopted as learning materials in a class, be suggested as "further readings" [24] or re-arranged in a repository with semantic capabilities [25].

At your convenience, please note that the demo is available online at the URL https://mc-unige.eu-gb.mybluemix.net (best viewed with Mozilla Firefox).

\section{CONCLUSIONS}

Using videos in e-learning activity has become popular for both the availability of a large amount of video content and the increase of MOOCs projects carried on by universities, which push their researchers to support their teaching activity by publishing videos instead of presentations, books or documents. The use of videos itself is not an advancement in the e-learning methodology, since it was commonly used in the past, even if delivered in different forms, e.g., video-tapes. Yet, next generation network and services, advanced semantic capabilities and the availability of the cognitive computing services joined with the big data allow the empowerment of videos towards a new generation of e-learning frameworks able to overcome the traditional drawbacks of this medium.

\section{ACKNOWLEDGMENTS}

We wish to thank Mr. Marco Placidi for his precious support in coding and setting up the demo application.

\section{REFERENCES}

[1] Alexa, "Top 500 sites on the web," Annual Report available at the URL: http://www.alexa.com/topsites, last visited, September, 2015.

[2] E. Garcia-Barriocanal, M.A. Sicilia, S. Sanchez-Alonso, M. Lytras, "Semantic annotation of video fragments as learning objects: a case study with YouTube videos and the Gene Ontology." Interactive Learning Environments, vol.19, no. 1, pp. 25-44, 2011.

[3] J.K. Tang, H. Xie, H., T.L. Wong, "A big data framework for early identification of dropout students in MOOC," in Technology in Education. Technology-Mediated Proactive Learning, Springer Berlin Heidelberg pp. 127-132, 2015.

[4] K.S. Hone and G.R. El Said, "Exploring the factors affecting MOOC retention: A survey study," Computers \& Education, vol. 98, pp. $157-$ $168,2016$.

[5] A. Elbadrawy, A. Polyzou, A. Ren, M. Sweeney, G. Karypis, H. Rangwala, "Predicting Student Performance Using Personalized Analytics," Computer, vol. 49, no. 4, pp. 61-69, 2016.

[6] K. Kobylinski, J. Bennett, N. Seto, G. Lo, F. Tucci, "Enterprise application development in the cloud with IBM Bluemix," in Proceedings of the $24^{\text {th }}$ Annual International Conference on Computer Science and Software Engineering (CASCON '14), pp. 276-279, 2014.

[7] J.E. Kelly, S. Hamm, "Smart machines: IBM's Watson and the era of cognitive computing." Columbia Business School Publishing, 2013.

[8] M. Coccoli, P. Maresca, L. Stanganelli, A. Guercio, "An experience of collaboration using a PaaS for the smarter university model," Journal of Visual Languages and Computing, vol. 31, no. 1, pp. 275$282,2015$.

[9] G. Booch, "The soul of a new Watson, IEEE Software," vol. 28, no. 4, pp. 9-10, 2011.

[10] S. Sudarsan, "Evolving to a new computing era: cognitive computing with Watson," Journal of computing sciences in colleges archive, vol. 29 , no. 4, pp. 4-4, 2014 
[11] A.M. Gliozzo, "IBM's Watson Jeopardy! computer shuts down humans in final game," IEEE Spectrum, vol.17, 2011.

[12] E.W. Brown, "Watson: the Jeopardy! challenge and beyond," in Proceedings of the $35^{\text {th }}$ International ACM SIGIR conference on research and development in information retrieval, 2012.

[13] E.W. Brown, "The Jeopardy! challenge and beyond," in Proceeding of the $12^{\text {th }}$ IEEE International conference on Cognitive Informatics \& Cognitive Computing (ICCI*CC), 2013.

[14] M.C. McCord, J.W. Murdock, B.K. Boguraev, "Deep parsing in Watson," IBM Journal of research and development, vol. 56, no. 3.4, 3:1, pp. 3:15, 2012.

[15] A. Lally, J.M. Prager, M.C. McCord, B.K. Boguraev, S. Pathwardhan, J. Fan, J. Fodor, J. Chu-Carrol, "Question analysis: how Watson reads a clue," IBM Journal of research and development, vol. 56, no. 3.4, pp. 2:1, 2:14, 2012.

[16] D. Ferrucci, A. Levas, S. Bagchi, G. Gondek, E.T. Mueller, "Watson: beyond Jeopardy!," Artificial Intelligence, 199-200, pp. 93-105, 2012.

[17] E.A. Epstein, M.I. Schor, B.S. Lyer, A. Lally, E.W. Brown, J. Cwiklik, "Making Watson fast," IBM Journal of Research and Development, vol. 56, no. 3.4, pp. 15:1-15:12, 2012.

[18] M. Coccoli, A. Guercio, P. Maresca, L. Stanganelli, "Smarter universities. A vision for the fast changing digital era," Journal of Visual Languages and Computing, vol.25, no.6, pp. 1003-1011, 2014.

[19] M. Coccoli, P.Maresca, L. Stanganelli, "Cognitive computing in education," Journal of e- Learning and Knowledge Society, vol.12, no.2, pp. 55-69, 2016.

[20] M. Coccoli, P. Maresca, L. Stanganelli, "The role of big data and cognitive computing in the learning process," Journal of Visual Languages and Computing, In press.

[21] M. Coccoli, S. Iacono, G. Vercelli, "Applying gamification techniques to enhance effectiveness of video-lessons", Journal of eLearning and Knowledge Society, vol. 11 no. 3, pp. 73-84, 2015.

[22] C. Perryer, N.A. Celestine, B. Scott-Ladd, C. Leighton, "Enhancing workplace motivation through gamification: Transferrable lessons from pedagogy," The International Journal of Management Education, vol. 14, no. 3, pp. 327-335, 2016.

[23] G. Adorni, S. Battigelli, D. Brondo, N. Capuano, M. Coccoli, S. Miranda, F. Orciuoli, L. Stanganelli, A.M. Sugliano, G. Vivanet, "CADDIE and IWT: two different ontology-based approaches to Anytime, Anywhere and Anybody Learning," Journal of e-Learning and Knowledge Society, vol. 6, no. 2, pp. 53-66, 2010.

[24] M. Coccoli, G. Vercelli, G. Vivanet, "Semantic Wiki: a collaborative tool for instructional content design," Journal of e-Learning and Knowledge Society, vol. 8, no. 2, pp. 113-122, 2012. 